$\xi=$ 离

\title{
Prevalence of avascular necrosis of femoral head in patients with cirrhosis of liver
}

\author{
Sanjeev Mahajan ${ }^{1}$, Daksh Gadi ${ }^{2}$ *, Rahul Gupta ${ }^{2}$, Anirudh Sharma ${ }^{3}$, \\ Premjeet Singh Thind ${ }^{4}$, Som Gupta ${ }^{5}$, Raminder Kaur ${ }^{6}$ \\ ${ }^{1}$ Director and Head of Department, Orthopaedics and Joint Replacement, Fortis Hospital, Ludhiana \\ ${ }^{2}$ Senior Resident, Department of orthopaedics and joint replacement, Fortis Hospital, Ludhiana \\ ${ }^{3}$ Resident, Department of orthopaedics, DMC, Ludhiana \\ ${ }^{4}$ Fellow, Department of Orthopaedics and joint replacement, Fortis Hospital, Ludhiana \\ ${ }^{5}$ Head of Department, Department of Physiotherapy, Fortis Hospital, Ludhiana \\ ${ }^{6}$ Physiotherapist, Department of Physiotherapy, Fortis Hospital, Ludhiana \\ *Corresponding author E-mail: dr_daksh@yahoo.com
}

\begin{abstract}
Background: Amongst the multiple etiologies implicated in the development of Avascular necrosis of the femoral head, literature regarding the association of cirrhosis of liver with osteonecrosis of femoral head is scanty and is correspondingly less studied. Considering a large number of patients with liver cirrhosis in this region of our country, this study was conducted to evaluate the association of cirrhosis as an independent risk factor in the development of osteonecrosis. To the best of our knowledge No such study has been done evaluating the same in this region.

Methods: This prospective analysis aimed to determine the prevalence of osteonecrosis of femoral head in patients with cirrhosis of liver, patients presenting to the hospital with established cirrhosis, diagnosed with help of ultrasonography, were evaluated for the occurance of Avascular necrosis of the femoral head.

Results: The prevalence of osteonecrosis of femoral head in patients with cirrhosis of liver was evaluated to be $1.2 \%$ in the present study Amongst this cirrhotic subgroup, the prevalence of osteonecrosis of femoral head in cirrhotic patients due to excessive alcohol consumption was calculated to be $1.1 \%$ and that of the cirrhotic patients independent of alcohol consumption came out to be $1.4 \%$.

Conclusion: Analysis of the results of the present study establishes cirrhosis of liver, the cause of which could be independent or related to alcohol consumption, as a risk factor for the development of osteonecrosis of femoral head. Significance of alcohol related cirrhosis resulting in AVN of the femoral head, though, is challenged by the present study.
\end{abstract}

Keywords: Avascular Necrosis; Liver Cirrhosis; Femur Head.

\section{Introduction}

Osteonecrosis of the femoral head also referred to as avascular necrosis, a disease with multiple etiologies that affects young and active adults, is characterized by decreased vascular supply to the subchondral bone of the femoral head, leading to destruction and collapse of the femoral head. (Aaron RK 2001). It was Koenig who first described this condition in 1888 , which he termed osteochondritis dissecans. Later in 1925, Haenish described the first case of osteonecrosis of femoral head.

Many conditions have been implicated in the development of this disease. These include trauma, steroid overuse, alcohol abuse, systemic lupus erythematosus, organ transplant, Caissons disease, sickle cell anemia, Perthes disease and coagulation disorder. (Asano T et al 2003) (Tektonidou MG, Moutsopoulos HM 2004) (Table 1)
Table 1: Main Conditions Associated with Atraumatic Osteonecrosis (Louis Solomon Et Al 2010)

\begin{tabular}{lll}
\hline Infections & $\bullet$ & Osteomyelitis \\
\hline Caisson disease & $\bullet$ & Septic arthritis \\
\hline Haemoglobinopathy & $\bullet$ & Sickle Cell Disease \\
\hline Storage disorders & $\bullet$ & Gauchers Disease \\
\hline & $\bullet$ & Familial thrombophilia \\
Coagulation disorders & $\bullet$ & Hypofibrinolysis \\
& $\bullet$ & Hypolipoproteinaemia \\
& & pura \\
\hline & $\bullet$ & Perthes' disease \\
& $\bullet$ & Cortisone administra- \\
& & tion \\
Other & $\bullet$ & Alcohol abuse \\
& $\bullet$ & Systemic Lupus Ery- \\
& & thematosus \\
& $\bullet$ & Pregnancy \\
& $\bullet$ & Anaphylactic shock \\
& $\bullet$ & Ionizing radiation \\
& $\bullet$ & Organ transplant \\
\hline
\end{tabular}


Regardless of the underlying etiology of osteonecrosis, the final outcome is apoptosis of osteoblasts and osteocytes. (Weinstein RS et al 2000) (Calder JD et al 2004) (Youm YS et al 2010). The pathophysiological model of osteonecrosis of femoral head proposes a multiple hit theory. (Schulte CM, Beelen DW 2004)

Unfortunately, out of the many risk factors only a few studies have been done to evaluate the association of individual risk factors in development of osteonecrosis of femoral head. The literature regarding the association of cirrhosis of liver with osteonecrosis of femoral head is scanty and is correspondingly less studied. (Hung $\mathrm{TH}$ et al 2011). Considering a large number of patients with liver cirrhosis in this region of our country and our hospital being a high volume centre for cirrhosis, this study was conducted to evaluate the association of cirrhosis as an independent risk factor in the development of osteonecrosis. No such study has been done evaluating the same in this region.

\section{Material and methods}

This was a prospective analysis of prevalence of osteonecrosis of femoral head in patients with cirrhosis of liver, conducted on patients undergoing treatment in our hospital. Patients with a prior diagnosis of cirrhosis of liver or patients who were diagnosed of cirrhosis were included in the study. Diagnosis was established using ultrasonography as the diagnostic modality for screening of patients along with laboratory tests for liver dysfunction. All age groups were included in the study.

Patients who were not willing to participate in the study and refused to give consent or had a history of trauma to the involved hip were excluded. The following algorithm (fig 2) was used for selection and evaluation of patients.

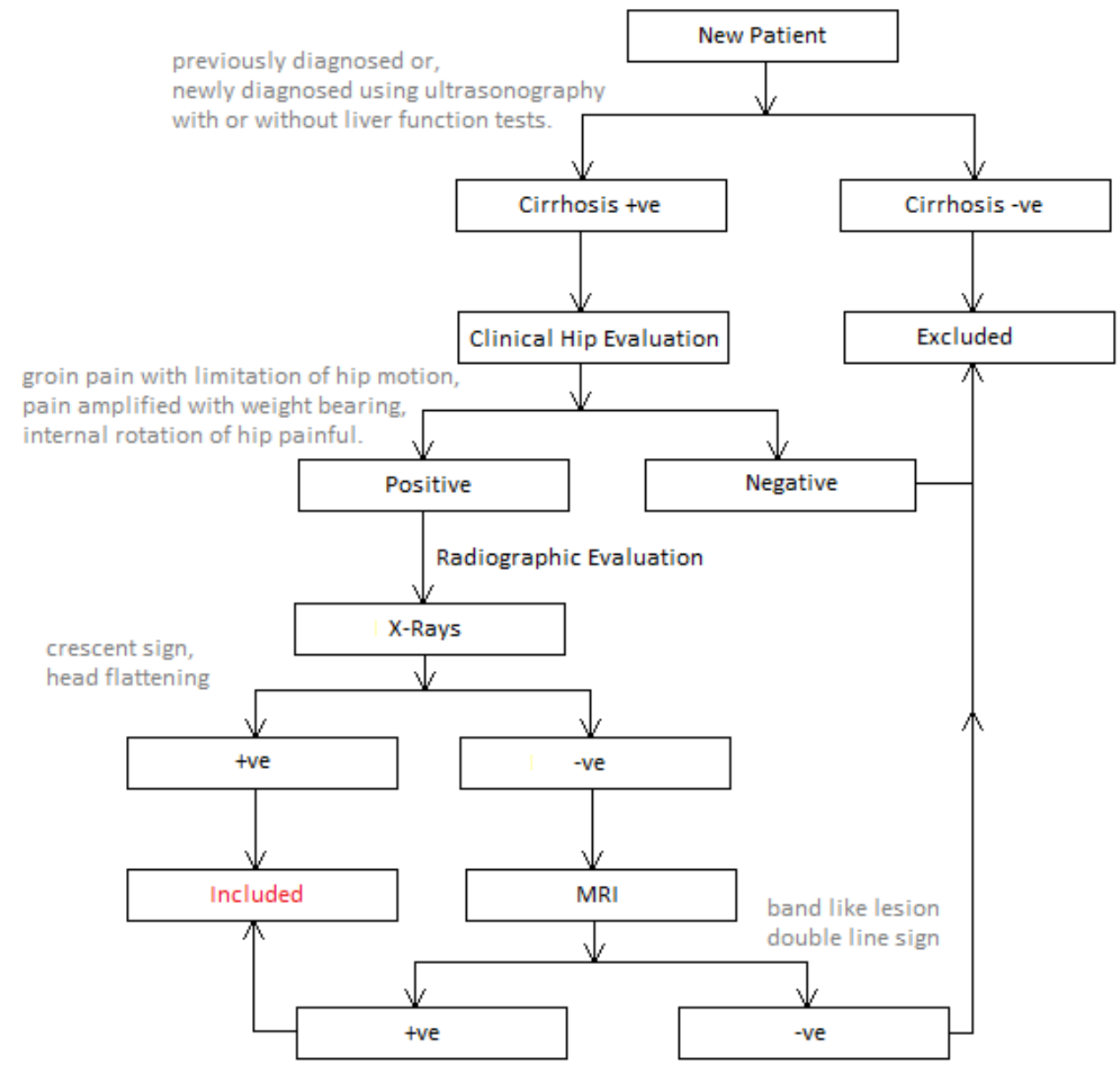

Fig. 2: Clinical Signs Were the Main Criteria of Selection in Our Study. This was followed with Subsequent Imaging Studies.

Patients were identified and enrolled for the study through proper clinical and radiographic evaluation. Clinically, medial thigh or groin pain with limitation of hip motion in cirrhotic patients raised our suspicion. Hip pain, which intensified with weight bearing and relieved with rest was noted. Range of motion was checked and if limited, particularly hip abduction and internal rotation, the patient was considered as a possible positive and was undertaken for radiographic evaluation.

Imaging studies were carried out using both the Ficat and Artlet staging and Association Research Circulation Osseous (ARCO) staging system. The former staging system was used for mass screening of patients. This included standard pelvis with both hips anteroposterior and lateral view radiographs with or without a magnetic resonance imaging (MRI). Patients who had signs of osteonecrosis on plain radiographs were taken as positive cases. For patients who had clinical symptoms with normal radiographs were planned for MRI of hip.

\section{Observation and results}

Amongst the 1050 patients that were evaluated, 737 patients met the inclusion criteria and were included in this study. The youngest patient was 22 years old and the eldest was 85 years old. The mean age was 50.82 years with a standard deviation of 10.753 . $621(84.2 \%)$ patients were between the age group of 36 to 65 years of age $(\mathrm{p}<0.001)$. Out of the total patients, $682(92.5 \%) \mathrm{pa}-$ tients were male and $55(7.5 \%)$ were female.

A large group of patients, to be exact $454(61.6 \%)$ patients, had cirrhosis related to alcohol abuse and the remaining 283 (38.4\%) had cirrhosis due to other causes $(\mathrm{p}<0.001)$ (Table 3$)$. 
Table 3: Distribution of Patients According to Etiology of Cirrhosis

\begin{tabular}{llll}
\hline Cirrhosis & No. of patients & Percent & P Value \\
\hline Alcohol related & 454 & 61.6 & \\
Other causes & 283 & 38.4 & $<0.001$ \\
Total & 737 & 100.0 & \\
\hline
\end{tabular}

Amongst all these patients, only $9(1.2 \%)$ were diagnosed with osteonecrosis of femoral head $(\mathrm{p}<0.001)$. Thus the prevalence of osteonecrosis of femoral head in patients with cirrhosis of liver was $1.2 \%$ in our study $(\mathrm{p}<0.001)$ (Table 4$)$. Amongst these 9 patients, $5(55.56 \%)$ had alcoholic liver disease and the remaining 4 $(44.44 \%)$ had cirrhosis related to other causes, of which $3(75 \%)$ were due to viral hepatitis. Of the 9 patients diagnosed with osteonecrosis of femoral head, 4 had unilateral joint involved, 3 $(33.33 \%)$ right and $1(11.11 \%)$ left and $5(55.56 \%)$ had bilateral joints involved.

Table 4: Fraction of Patients Diagnosed with Osteonecrosis

\begin{tabular}{llll}
\hline Osteonecrosis & No. of patients & Percent & P Value \\
\hline No & 728 & 98.8 & \\
Yes & 9 & 1.2 & $<0.001$ \\
Total & 737 & 100.0 & \\
\hline
\end{tabular}

Thus the prevalence of osteonecrosis of femoral head in cirrhotic patients due to excessive alcohol consumption was calculated to be $1.1 \%$ and that of the cirrhotic patients independent of alcohol consumption came out to be $1.4 \%$ in the present study (table 5).

Table 5: Proportion of Patients Diagnosed with Osteonecrosis in Cirrhotic Subgroup

\begin{tabular}{llll}
\hline Cirrhosis & No. of patients & Osteonecrosis & Percent \\
\hline Other causes & 283 & 4 & $1.4 \%$ \\
Alcohol related & 454 & 5 & $1.1 \%$ \\
\hline
\end{tabular}

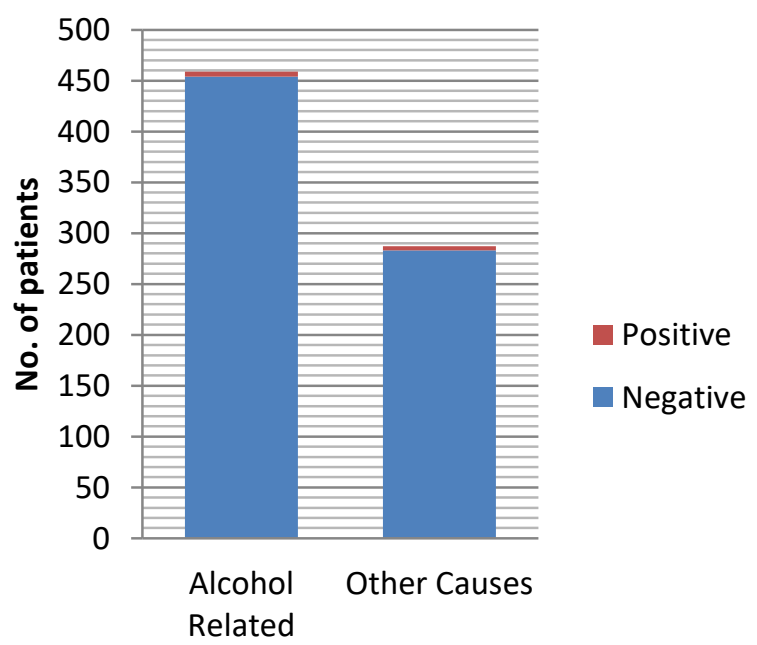

Cirrhotic Sub-Groups

\section{Discussion}

Osteonecrosis of femoral head traditionally is managed by the orthopaedic team. Numerous studies looking right from etiology to treatment of this disease have been undertaken. Over a period of time a lot of etiological factors have been implicated to cause osteonecrosis of femoral head. The present study evaluates the prevalence of osteonecrosis of femoral head in patients with cirrhosis of liver.

Cirrhosis is commonly seen in patients with alcohol abuse. Apart from alcohol, there are other etiologies that cause cirrhosis. Amongst these, viral hepatitis is emerging as an important leading cause. Thus, the most common etiologies of cirrhosis are alcohol abuse and viral hepatitis. The same was observed in the present study. However, it is still not clear whether cirrhosis of liver in itself is associated with osteonecrosis of femoral head, or, are there other adjuvant factors that cause this disease.

In the present study, most cases of cirrhosis were due to alcohol abuse $(61.3 \%)$. It was also observed that the prevalence of osteonecrosis of femoral head in patients with cirrhosis independent of alcohol abuse was comparable to that of patients with cirrhosis due to alcohol abuse. This was an important finding as there have been many studies that implicate alcohol abuse as an important risk factor in the development of osteonecrosis, on the contrary, the findings of present study refutes the same and questions the involvement of alcohol as an independent and important risk factor resulting in osteonecrosis of femoral head.

In the present study, an increased risk of osteonecrosis of femoral head was also noticed in patients with male sex and excessive alcohol consumption, as is seen in the Indian population. Hirota et al. have also reported similar results in patients with excessive alcohol intake, cigarette smoking along with their occupational status with the risk developing idiopathic osteonecrosis of the femoral head. (Hirota Y et al 1993). Fukushima et al. in 2010 conducted a nationwide epidemiologic survey of idiopathic osteonecrosis of the femoral head with findings comparable to this study. (Fukushima Wet al 2010).

Despite the fact that many associated factors that have been proposed, the pathogenesis of the disease is not well understood. Impairment of the vascular supply to the femoral neck is considered as an important mechanism for osteonecrosis of femoral head. (Jones LC, Hungerford DS 2004). This occurs when the local blood supply to the femoral head is severed, commonly seen after trauma. Osteonecrosis of femoral head may be caused by other mechanisms other than impairment of vascular supply. Some authors have proposed venous stasis and retrograde arteriolar stoppage as an important mechanism. While the possibility of intravascular thrombosis and compression of capillaries and sinusoids by marrow swelling as a possible mechanism in the pathogenesis of osteonecrosis have also been debated. The end result of this vascular insult, results in death of osteocytes and eventual collapse of the articular surface.

Although evidence regarding cirrhosis as a possible risk factor in the etiopathogenesis of osteonecrosis of femoral head is lacking but there are many studies which indicate the prevalence of bone mineral disorders in patients with cirrhosis. Certain studies have shown increased bone resorption and decreased bone formation in patients with chronic liver disease. (Gallego-Rojo FJ et al 1998) (Crosbie OM et al 1999)

Patients with cirrhosis of liver have anomalies of calcium and vitamin D metabolism. Vitamin D undergoes 25 -hydroxylation in the liver tissue, a process that is affected by advanced liver disease. Various studies have shown low serum 25-hydroxyvitamin $\mathrm{D}$ levels in individuals affected by chronic liver disease. (Crawford BA et al 2006) (George $J$ et al 2009) Reduced tissue sensitivity to circulating vitamin $\mathrm{D}$ due to altered vitamin $\mathrm{D}$ receptor genotypes may play a role. Vitamin $\mathrm{K}$ is an essential cofactor for osteoblasts to synthesize osteocalcin - bone matrix protein. A decreased osteocalcin level in patients with cirrhosis means reduced bone formation. Thus, Vitamin K deficiency contributes to osteopenia. (Nishiguchi S et al 2001)

Many studies have demonstrated the role of insulin like growth factor 1 (IGF-1), interlukin 6 (IL-6), osteoprotegerin (OPG) and tumor necrosis factor $\alpha(\mathrm{TNF}-\alpha)$ in the development of hepatic osteodystrophy. A decreased IGF-1 level in patients with liver cirrhosis may contribute to the development of osteoporosis. Increased TNF- $\alpha$ and IL- 6 levels in patients with cirrhosis can cause chronic joint inflammation and lead to arthritis. (McInnes IB, Schett G. 2007) (Smolen JS et al 2008). IL-6 can induce osteoclastogenesis and bone resorption (Liu XH et al 2005) (Wong PK et al 2006) (De Benedetti $F$ et al 2006). Various studies has shown that the OPG is high in patients with chronic liver disease compared to control subjects, which shows that there is ligand consumption and overexpression of OPG that activates osteoclastic activity. (Moschen AR et al 2005) (González-Calvin JL et al 2009). Cirrhosis of liver is associated with coagulation dysfunction along 
with thrombocytopenia which has been proved to be a risk factor in development of osteonecrosis of femoral head.

Wiest et al. in 2011 evaluated the hepatic removal of IL-6 in patients with cirrhosis and proved impaired hepatic clearance of IL-6 in cirrhotic patients (Wiest $\mathrm{R}$ et al 2005). They hypothesized that high osteoclastogenesis induced by raised IL-6 levels to be the most important reason for atraumatic osteonecrosis of femoral head in cirrhotic patients. This may cause bone resorption and subsequent collapse of the subchondral bone. There are many factors that cause bone disorders in cirrhotic patients but further studies are required to prove more evidence to support cirrhosis as an individual risk factor in the pathogenesis of osteonecrosis of femoral head.

This study however has certain limitations. First, the database we selected does not include complete information that may be relevant to our study, such as smoking, the use, dosage and duration of steroid, any of which can cause osteonecrosis of femoral head. Secondly, the true frequency of osteonecrosis of femoral head may be underestimated in this study, because not all patients with osteonecrosis of femoral head require hospital admission. However, hospitalization for osteonecrosis of femoral head would be a marker for a disabling or more severe form of the disease, which may have more important clinical significance.

Third, as only radiographs were employed in mass screening of patients as per our study algorithm and MRI was only done for patients with normal radiographs and positive clinical findings, we might have missed the disease in its early stages.

Fourth, as our study only conducted the prevalence of the disease in cirrhotic patients, we didn't have a control group to compare our findings with non-cirrhotic patients. Considering the results of our study in wholesome and analyzing the above said limitations, it can be readily quoted that cirrhosis of liver occurring in the absence of alcohol abuse can be considered as an important etiopathogenic factor in causing osteonecrosis of femoral head. However, further studies are required to strongly establish this fact. Thus, cirrhosis of liver can be considered as an etiological factor independent of alcohol consumption in the pathogenesis of osteonecrosis of femoral head.

\section{Conclusion}

This study intended to find the prevalence of osteonecrosis of femoral head in patients with cirrhosis of liver. As per this study, the prevalence of osteonecrosis of femoral head in patients with cirrhosis of liver is $1.2 \%$ and it can be concluded that cirrhosis of liver is a risk factor in the development of osteonecrosis of femoral head. Alcohol is an established risk factor for osteonecrosis of femoral head. Hence any patient with cirrhosis of liver, the cause of which could be independent or related to alcohol consumption should always be considered a risk factor for the development of osteonecrosis of femoral head. Thus, physicians should be aware of the importance of evaluating hip pain in cirrhotic patients.

After conducting such a study the question that came across our minds was that whether cirrhosis secondary to alcohol abuse was really a significant risk factor for development of osteonecrosis of femoral head. This of course is a matter of discussion. Similarly, cirrhosis independent of alcohol abuse should be considered as an equally important risk factor.

\section{References}

[1] Aaron RK, Ciombor DM. Coagulopathies and osteonecrosis. Curr Opin Orthop 2001; $12: 378-383$ http://dx.doi.org/10.1097/00001433-200110000-00003.

[2] Asano T, Takahashi KA, Fujioka M, Inoue S, Okamoto M, Sugioka $\mathrm{N}$ et al. A polymorphism decreased the risk for steroid-induced osteonecrosis of the femoral head after kidney transplantation. Pharmacogenetics 2003 ;

13:675-682 http://dx.doi.org/10.1097/00008571-200311000-00003.
[3] Tektonidou MG, Moutsopoulos HM. Immunologic factors in the pathogenesis of osteonecrosis. Orthop Clin North Am 2004; 35:259-263. http://dx.doi.org/10.1016/j.ocl.2004.02.003.

[4] Louis Solomon, David Warwick, Selvadurai Nayagam, 2010 Apley's System of Orthopaedics and Fractures, ninth edition, Hodder Arnold, London. http://dx.doi.org/10.1201/b13422.

[5] Calder JD, Buttery L, Revell PA, Pearse M, Polak JM. Apoptosis--a significant cause of bone cell death in osteonecrosis of the femoral head. J Bone Joint Surg Br. 2004; 86:1209-1213. http://dx.doi.org/10.1302/0301-620X.86B8.14834.

[6] Weinstein RS, Nicholas RW, Manolagas SC. Apoptosis of osteocytes in glucocorticoid-induced osteonecrosis of the hip. J Clin Endocrinol Metab. 2000; 85:2907-2912. http://dx.doi.org/10.1210/jc.85.8.2907.

[7] Youm YS, Lee SY, Lee SH. Apoptosis in the osteonecrosis of the femoral head. Clin Orthop Surg. 2010; 2:250-255 http://dx.doi.org/10.4055/cios.2010.2.4.250.

[8] Schulte CM, Beelen DW: Avascular osteonecrosis after allogeneic hematopoietic stem-cell transplantation: diagnosis and gender matter. Transplantation 2004, 78:1055-1063. http://dx.doi.org/10.1097/01.TP.0000138026.40907.38.

[9] Hung TH, Hsieh YH, Tsai CC, Tseng CW, Tseng KC, Tsai CC. Is liver cirrhosis a risk factor for osteonecrosis of the femoral head in adults? A population-based 3-year follow-up study. Intern Med. 2011; 50(21):2563-8 http://dx.doi.org/10.2169/internalmedicine.50.5952.

[10] Hirota Y, Hirohata T, Fukuda K, Mori M, Yanagawa H, Ohno Y et al. Association of alcohol intake, cigarette smoking, and occupational status with the risk of idiopathic osteonecrosis of the femoral head. Am J Epidemiol. 1993; 137 (5):530-538.

[11] Fukushima W, Fujioka M, Kubo T, Tamakoshi A, Nagai M, Hirota Y. Nationwide epidemiologic survey of idiopathic osteonecrosis of the femoral head. Clin Orthop Relat Res. 2010; 468: 2715-2724. http://dx.doi.org/10.1007/s11999-010-1292-x.

[12] Jones LC, Hungerford DS. Osteonecrosis: etiology, diagnosis, and treatment. Curr Opin Rheumatol. 2004; 16: 443-449. http://dx.doi.org/10.1097/01.moo.0000127829.34643.fd.

[13] Gallego-Rojo FJ, Gonzalez-Calvin JL, Muñoz-Torres M, Mundi JL, Fernandez-Perez R, Rodrigo-Moreno D. Bone mineral density, serum insulin-like growth factor I, and bone turnover markers in viral cirrhosis. Hepatology. 1998; 28:695-699. http://dx.doi.org/10.1002/hep.510280315.

[14] Crosbie OM, Freaney R, McKenna MJ, Hegarty JE. Bone density, vitamin $\mathrm{D}$ status, and disordered bone remodeling in end-stage chronic liver disease. Calcif Tissue Int. 1999; 64:295-300. http://dx.doi.org/10.1007/s002239900622.

[15] Crawford BA, Labio ED, Strasser SI, McCaughan GW. Vitamin D replacement for cirrhosis-related bone disease. Nat Clin Pract Gastroenterol Hepatol. 2006; 3:689-699. http://dx.doi.org/10.1038/ncpgasthep0637.

[16] George J, Ganesh HK, Acharya S, Bandgar TR, Shivane V, Karvat A et al. Bone mineral density and disorders of mineral metabolism in chronic liver disease. World J Gastroenterol. 2009; 15:35163522. http://dx.doi.org/10.3748/wjg.15.3516.

[17] Nishiguchi S, Shimoi S, Kurooka H, Tamori A, Habu D, Takeda T et al. Randomized pilot trial of vitamin K2 for bone loss in patients with primary biliary cirrhosis. J Hepatol. 2001; 35:543-545. http://dx.doi.org/10.1016/S0168-8278(01)00133-7.

[18] McInnes IB, Schett G. Cytokines in the pathogenesis of rheumatoid arthritis. Nat Rev Immunol 2007; 7: 429-442. http://dx.doi.org/10.1038/nri2094.

[19] Smolen JS, Beaulieu A, Rubbert-Roth A, et al. Effect of interleukin-6 receptor inhibition with tocilizumab in patients with rheumatoid arthritis (OPTION study): a double-blind, placebocontrolled, randomised trial. Lancet 2008; 371: 987-997. http://dx.doi.org/10.1016/S0140-6736(08)60453-5.

[20] Wong PK, Quinn JM, Sims NA, van Nieuwenhuijze A, Campbell IK, Wicks IP. Interleukin-6 modulates production of T lymphocytederived cytokines in antigen-induced arthritis and drives inflammation-induced osteoclastogenesis. Arthritis Rheum 2006; 54: 158168. http://dx.doi.org/10.1002/art.21537.

[21] De Benedetti F, Rucci N, Del Fattore A, et al. Impaired skeletal development in interleukin-6-transgenic mice: a model for the impact of chronic inflammation on the growing skeletal system. Arthritis Rheum 2006; 54: 3551-3563. http://dx.doi.org/10.1002/art.22175.

[22] Liu XH, Kirschenbaum A, Yao S, Levine AC. Cross-talk between the interleukin- 6 and prostaglandin E(2) signaling systems results in enhancement of osteoclastogenesis through effects on the osteoprotegerin/ receptor activator of nuclear factor-\{kappa\}B (RANK) lig- 
and/RANK system. Endocrinology 2005; 146: 1991-1998. http://dx.doi.org/10.1210/en.2004-1167.

[23] Moschen AR, Kaser A, Stadlmann S, Millonig G, Kaser S, Mühllechner $\mathrm{P}$ et al. The RANKL/OPG system and bone mineral density in patients with chronic liver disease. J Hepatol. 2005; 43:973-983. http://dx.doi.org/10.1016/j.jhep.2005.05.034.

[24] González-Calvin JL, Mundi JL, Casado-Caballero FJ, Abadia AC, Martin-Ibañez JJ. Bone mineral density and serum levels of soluble tumor necrosis factors, estradiol, and osteoprotegerin in postmenopausal women with cirrhosis after viral hepatitis. J Clin Endocrinol Metab. 2009; 94:4844-4850. http://dx.doi.org/10.1210/jc.2009$\underline{0835}$.

[25] Wiest R, Weigert J, Wanninger J, et al. Impaired hepatic removal of interleukin-6 in patients with liver cirrhosis. Cytokine 2011; 53:178-183. http://dx.doi.org/10.1016/j.cyto.2010.06.013 